\title{
10q22.3q23.3 microduplication syndrome
}

INSERM

\section{Source}

INSERM. (1999). Orphanet: an online rare disease and orphan drug data base.

10q22.3q23.3 microduplication syndrome. ORPHA:276422

$10 q 22.3 q 23.3$ microduplication syndrome is a rare, chromosomal anomaly characterized by variable clinical features that may include developmental delay, mild intellectual disability and dysmorphic facial features. In some cases, microcephaly, growth retardation and congenital heart defects have been reported. 